\title{
A new method for the preparation of cobalt nanoferrite/graphene composite
}

\author{
Shaimaa A.Habib ${ }^{1,3}$, Samia A. Saafan1,Talaat M. Meaz ${ }^{1}$, Tarek \\ Hussein $^{2}$, Hussein A. Motaweh ${ }^{3}$, P. Knoll ${ }^{4}$ \\ ${ }^{1}$ Physics Department, Faculty of Science, Tanta University, Tanta 31527, \\ Egypt \\ ${ }^{2}$ Physics Department, Faculty of Science, Cairo University, Cairo, Egypt \\ ${ }^{3}$ Physics Department, Faculty of Science, Damnhour University, \\ Damanhour, Egypt \\ ${ }^{4}$ Institute of Physics, Karl-Franzens-University, Universitätsplatz 5, A- \\ 8010 Graz, Austria
}

The current work reports the preparation of graphene and cobalt nanoferrite /graphene composite by using Plasma Enhanced Chemical Vapor Deposition (PECVD). At first, the cobalt ferrite nanoparticles have been prepared via co-precipitation method. Then the graphene has been deposited by PECVD on one pure $\mathrm{Cu}$ substrate and on another $\mathrm{Cu}$ substrate coated by the slurry of Co nanoferrite. Raman spectroscopy has been used to ensure the formation of graphene in both graphene and composite samples. The Raman spectrum has also confirmed the existence of the ferrite in the composite. The scanning electron microscope images have revealed a quite uniform porous surface structure of graphene in both samples but the homogeneity has been enhanced in the composite. Moreover, the existence of ferrite slurry layer underneath the graphene has reduced surface defects too. This new successful method of preparing cobalt nanoferrite /graphene composite on a Cu substrate with the aid of PECVD may find a very useful application in producing high performance electrodes in batteries or supercapacitors. Therefore, measuring the electrochemical performance of this prepared composite as an electrode will be our near future work. 


\section{Introduction}

Since its successful isolation from graphite in 2004, Graphene has drawn attention to the world of 2D materials [1]. From a just unknown material it has become a super star in several fields of technology and academic research. Unique properties of graphene, such as high thermal conductivity, high current density, super hydrophobicity, ballistic transport, and optical transmittance have been recorded [2]. Graphene can be simply defined as a single layer of sp2 bonded carbon atoms in a hexagonal lattice or two interleaving triangular lattices providing graphene's stability [3].

Ferrites are basically ferrimagnetic complex oxides possessing very high resistivity and high magnetic susceptibility. The saturation magnetization of ferrites is less than half of ferromagnetic alloys, nevertheless they possess advantages such as lower price, greater heat resistance, higher corrosion resistance and safe application at high ac frequencies [4].

Commercial application of ferrites had begun since 1950's in electronics, communications and microwave devices. Magnetic and dielectric properties are strongly dependent on particle size, thus synthesizing different size particle ferrites may serve to tailor the materials for desired applications [5].

Recently, nanoferrite/graphene composites have emerged as new materials for many important applications, specially for energy storage. These composites may provide promising candidates for both supercapacitors [6]. , and battery systems [7].

A nanoferrite/graphene composite may provide a relatively large surface to volume ratio, which would be suitable for some important interactions and reactions. Moreover, such heteroarchitectures may display unique electrochemical behavior generated by the magnetic nature of ferrites and the electrical conductivity of graphene sheets [8]. Besides, it is now well known that graphene and graphene-based hybrids can be considered as potential candidates for replacing Si-based technologies due to their extraordinary properties, being less toxic and biodegradable [9].

The aim of the present work is to synthesize graphene and cobalt nanoferrite /graphene composite on $\mathrm{Cu}$ substrates with the aid of Plasma Enhanced Chemical Vapor Deposition (PECVD), to confirm their formation by using the non-destructive Raman spectroscopy and finally to detect the surface structure 
of graphene in both samples and the difference between them by using the scanning electron microscope.

\section{Experimental}

\subsection{Preparation of Cobalt ferrite $\left(\mathrm{CoFe}_{2} \mathrm{O}_{4}\right)$ in aqueous medium:}

Ferrites nanoparticles can be synthesized by various ways, the most common method is the chemical co-precipitation of Fe salts with the addition of a hydroxide base. This method allows the preparation of magnetic nanoparticles in a simple way with the ability of controlling size and shape and thus it is a very appropriate method to use in various applications [10-11].

$\mathrm{CoFe} 2 \mathrm{O} 4$ nanoparticles had been prepared by alkaline co-precipitation of ferric and cobalt salts in aqueous solution as follows:

1. $1.0 \mathrm{ml}$ of Cobalt nitrate (anhydrous (CoNH3) SIGMA ALDERISH Chemicals, 99.99\%) and $4.0 \mathrm{ml}$ of ferric nitrate (anhydrous (FeNH3) SIGMA ALDERISH Chemicals, 99.99\%) have been mixed in a $100 \mathrm{ml}$ beaker equipped with a magnetic bar.

2. During vigorous stirring, a $50.0 \mathrm{ml}$ of $1 \mathrm{M}$ Ammonium hydroxide ((NH4 OH), SIGMA ALDERISH, 99\%) has been added drop-wise to the reaction mixture (at a temperature of $80 \mathrm{o} \mathrm{C}$ ) to adjust the $\mathrm{PH}$ at about 13 , and stirred for two hours. A very black precipitate has been formed which is the Co ferrite.

3. The stirrer has been switched off and the precipitate has been collected by using a medium strength magnet.

4. The product has been washed many times with pure distilled water and then dried at $60{ }^{\circ} \mathrm{C}$.

\subsection{Preparation and synthesis of graphene by PECVD:}

The growth process of graphene on copper, the preferred substrate, has been performed in the reaction chamber illustrated in Fig. 1 . The copper is preferred because of the poor solubility of carbon in copper, even at high temperatures (e.g. $1000{ }^{\circ} \mathrm{C}$ ). The formation of graphene can be described as a surface-mediated process.. The challenge of sample preparation is to define the right conditions in order to obtain a clean structure. To produce clean graphene on a relative big surface, the commonly used method is CVD. In that case, carbon atoms from a gas mixture of $\mathrm{CH} 4$ and $\mathrm{H} 2$ will be deposited on the substrate. The CVD process may strongly be enhanced by a plasma which dissociates methane molecules at significant lower temperatures (e.g. $500{ }^{\circ} \mathrm{C}$ ). This is now Plasma Enhanced CVD. 
The growth of graphene in the reaction chamber starts right after the hydrocarbon dissociation (in the plasma and on the surface) and stops immediately when the supply of hydrocarbon is cut off [12].

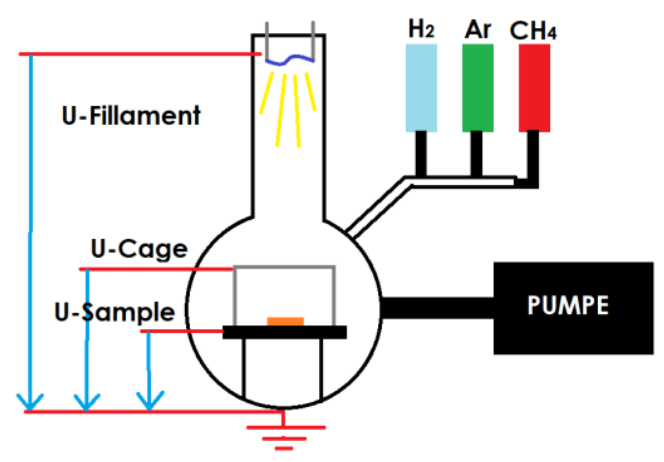

Figure (1) Schematic construction of the reaction chamber

\subsection{Preparation of cobalt nano-ferrite/ graphene composite:}

A slurry of the cobalt ferrite nanoparticles with dimethyl formamide (DMF) has been prepared and casted on a $\mathrm{Cu}$ substrate and by using the above mentioned plasma enhanced chemical vapor deposition method, layers of graphene have been deposited on the surface of the ferrite. This new method, of preparing such a composite has not been reported in literature before, as far as the authors know.

\subsection{Characterization of the samples}

The samples have been characterized by using XRD, Raman spectroscopy and SEM imaging at Graz University, Austria.

\section{Results and discussion}

\subsection{XRD of Co nano-ferrite}

Figure (2) shows the XRD of $\mathrm{CoFe} 2 \mathrm{O} 4$ nanoparticles prepared by the coprecipitation method.

The XRD pattern confirms the formation of the required face-centered cubic ferrite with a lattice parameter $\mathrm{a}=8.391 \AA$. The standard XRD peaks of ferrites 
indicate the planes [220], [311], [222], [400], [331], [422], [511], and [440] in agreement literature [13] .

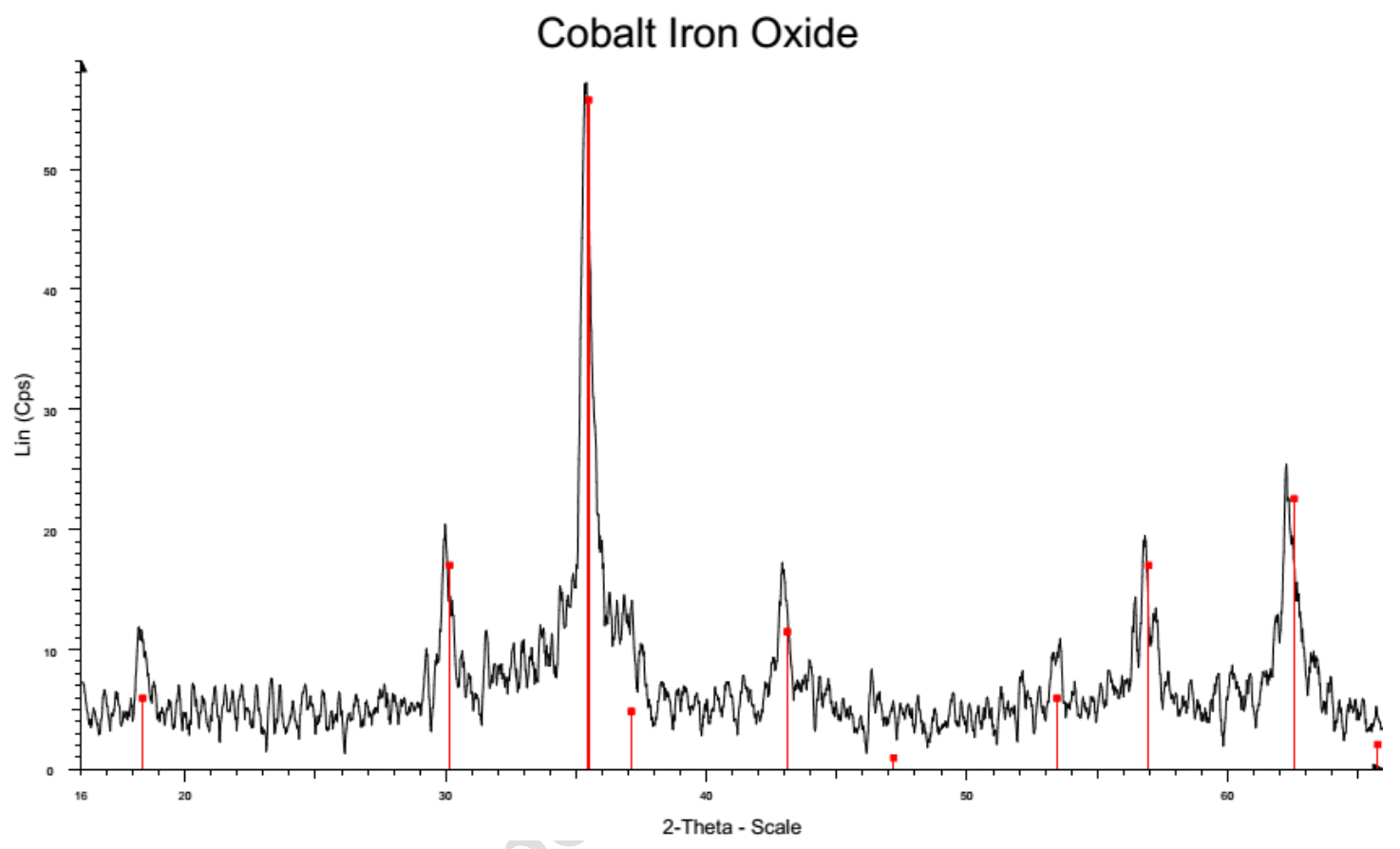

Figure (2) XRD of $\mathrm{CoFe} 2 \mathrm{O} 4$ nanoparticles

\subsection{Raman spectroscopy}

A laser irradiates the sample and its interaction with molecular vibrations, phonons or other excitations generates an infinitesimal amount of Raman scattered light detected by a CCD detector. The dominant Rayleigh scattered light is rejected using a filter and only the Stokes (or Anti-Stokes) Raman scattering are recorded. As it is well known that, Raman spectroscopy is used for identifying substances, polymorphs or tracking changes in molecular structures, it has played an important role in the structural characterization of graphitic materials and has been used to determine the number of layers of multilayer graphene samples and the quality of such samples. Raman spectra of multilayer graphene samples usually show three characteristic peaks denoted D $\left(1350 \mathrm{~cm}^{-1}\right), \mathrm{G}(1580$ $\left.\mathrm{cm}^{-1}\right)$ and $2 \mathrm{D}\left(2690 \mathrm{~cm}^{-1}\right)$ [14]. 
Moreover, it is worth mentioning that graphene can be differentiated easily from graphite by Raman spectroscopy, since graphene would have aa higher relative intensity in the 2D band compared to that of graphite along with a $2 \mathrm{D}$ band shift towards lower wave number compared to that of graphite Moreover, it is worth mentioning that graphene can be differentiated easily from graphite by Raman spectroscopy, since graphene would have aa higher relative intensity in the $2 \mathrm{D}$ band compared to that of graphite along with a $2 \mathrm{D}$ band shift towards lower wave number compared to that of graphite [15].

Figure (3) confirms the formation of graphene in the first sample i.e. graphene deposited on a pure $\mathrm{Cu}$ substrate.

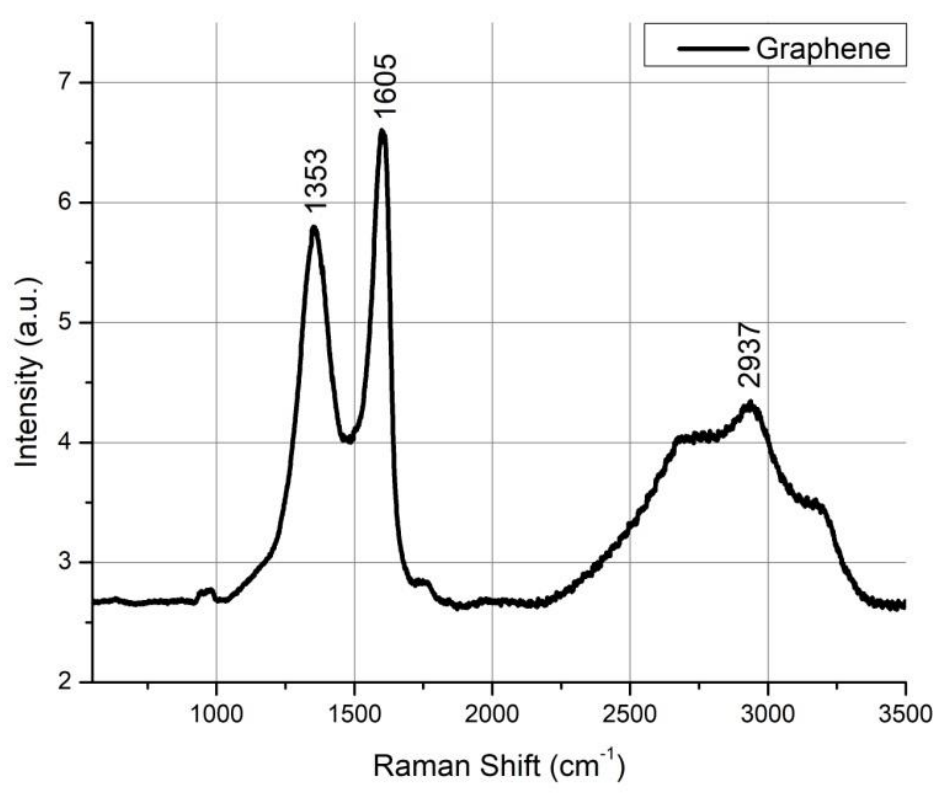

Figure (3) Raman spectrum of graphene deposited on a Cu substrate by PECVD 


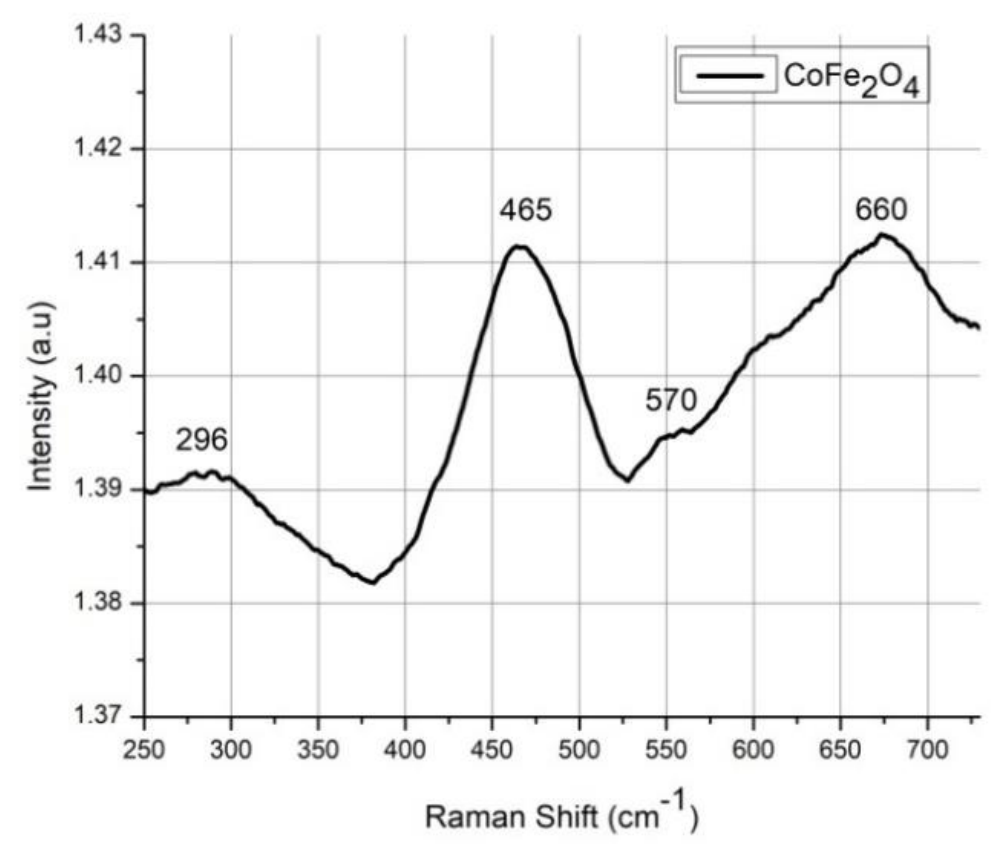

Figure (4): Raman spectrum of Co ferrite slurry casted on a $\mathrm{Cu}$ substrate

Raman spectra of ferrite samples usually show characteristic peaks around $198,297,461,558,604$ and $680 \mathrm{~cm}^{-1}$ [16]. Figure (4) confirms the formation of $\mathrm{Co}$ ferrite casted on the second $\mathrm{Cu}$ substrate before graphene deposition. By performing the fitting of the Raman spectra displayed, it would be possible to observe Raman bands at 296, 300, 465, 570, and 660 in fair agreement with literature. 


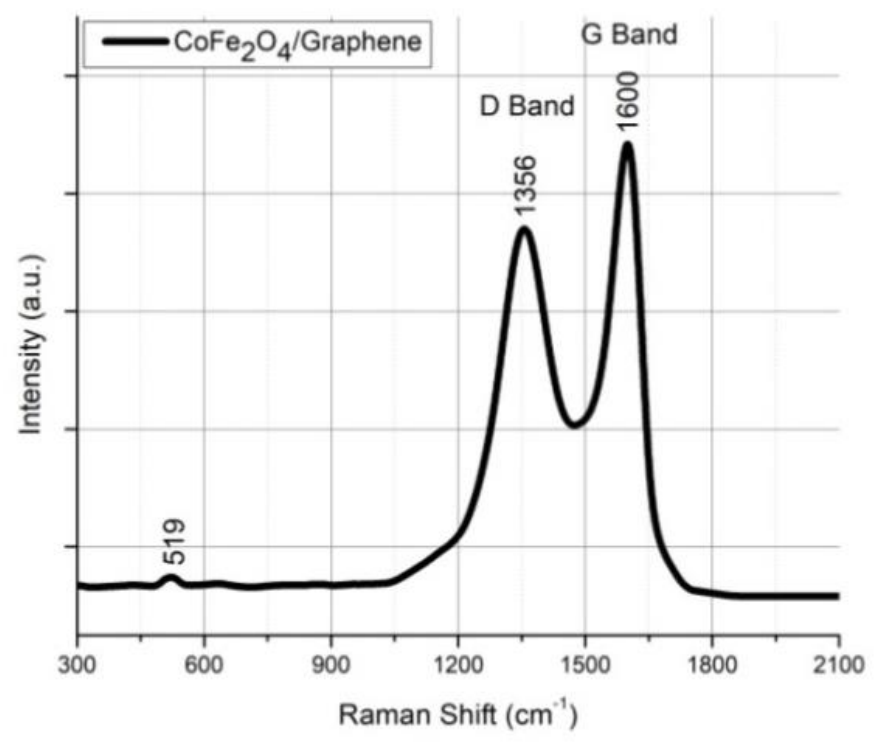

Figure (5): Raman spectrum of graphene /Co nanoferrite composite ( the graphene is deposited by PECVD on the $\mathrm{Cu}$ substrate coated by Co ferrite slurry)

In Figure (5) Raman spectrum of graphene /Co nanoferrite composite- where the graphene is deposited by PECVD on the $\mathrm{Cu}$ substrate coated by Co ferrite slurry- shows bands at 519, 1356 and 1600, in agreement with literature [17]. Unfortunately, the spinel $\mathrm{CoFe} 2 \mathrm{O} 4$ phase cannot been clearly seen due to the large amount of graphene [17]. For CoFe2O4/Graphene composite sample, the intensity ratio of D-band to G-band ID/IG less than the ratio for Graphene. This indicates that the presence of $\mathrm{CoFe} 2 \mathrm{O} 4$ decreased the defects.

\subsection{SEM spectroscopy}

The existence of ferrite slurry on the substrate has reduced defects in the deposited graphene surface as reported in literature [18]. This has been evidenced in SEM images.

Figures (6) and (7) show the SEM images of the graphene sample and the composite sample respectively with the same magnification scale. The surface defects have been obviously reduced in Figure (7). 


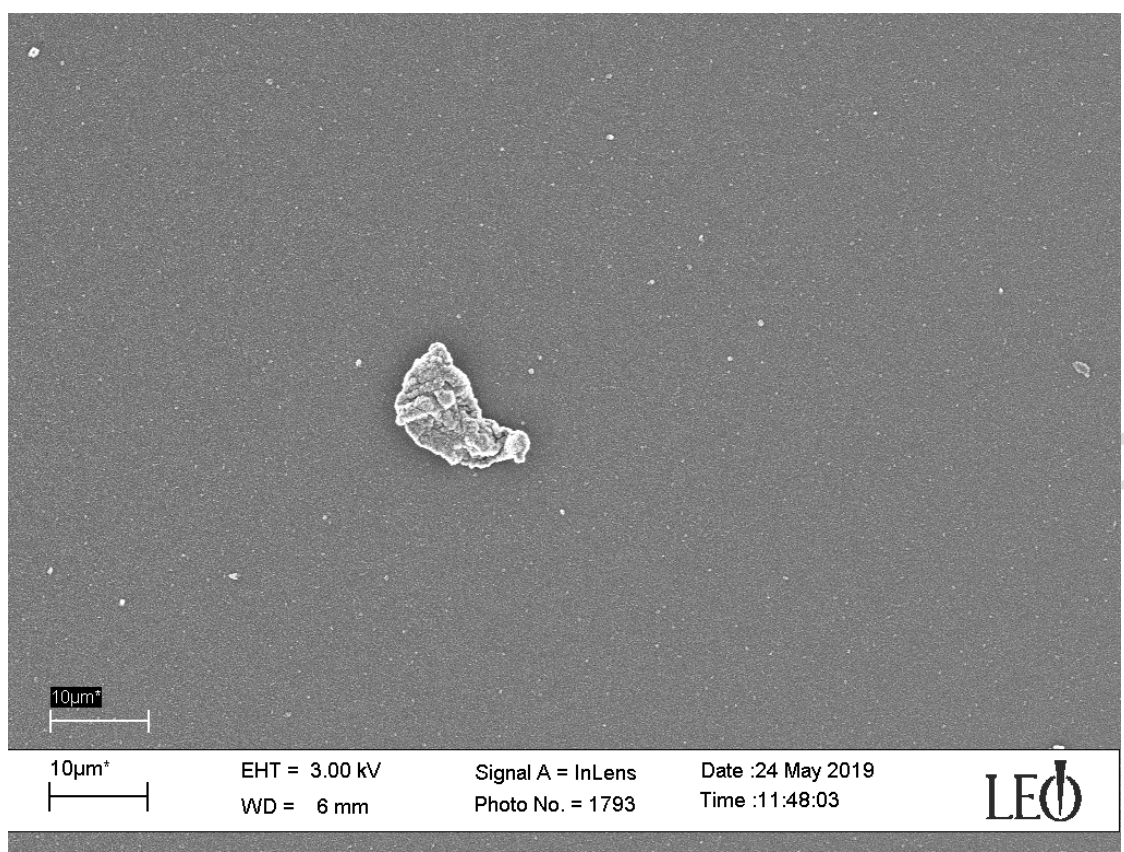

Figure (6) SEM image of graphene deposited on a Cu substrate (some defects in the surface structure are shown)

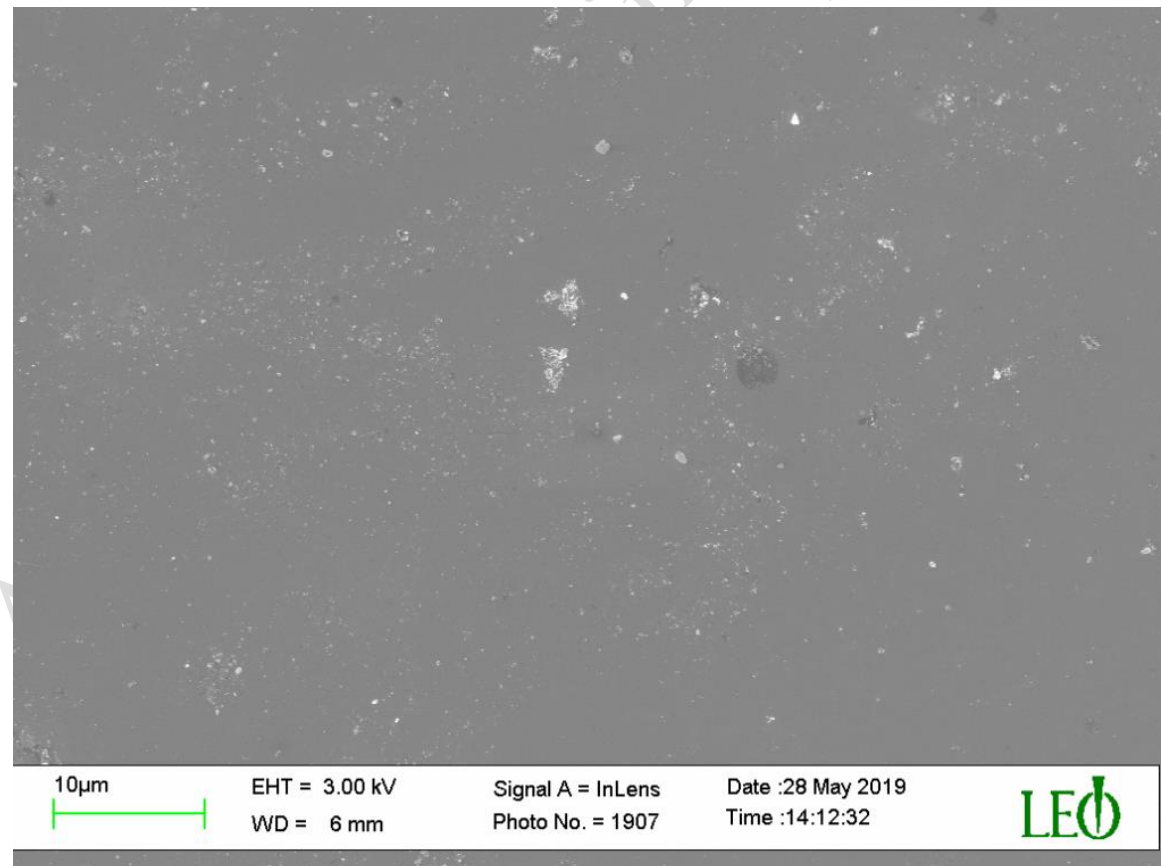

Figure (7) SEM image of graphene deposited on the $\mathrm{Cu}$ substrate coated by a slurry of Co nanoferrite (Less defects and more homogeneity are observed) 
Figures (8) and (9) show more obviously the effect of the existence of the Co nanoferrite on the formation of graphene. In Figure (9), the layer of nanoferrite underneath the graphene has greatly enhanced homogeneity and regularity of the graphene formation on the surface. Better distribution of pores is observed.

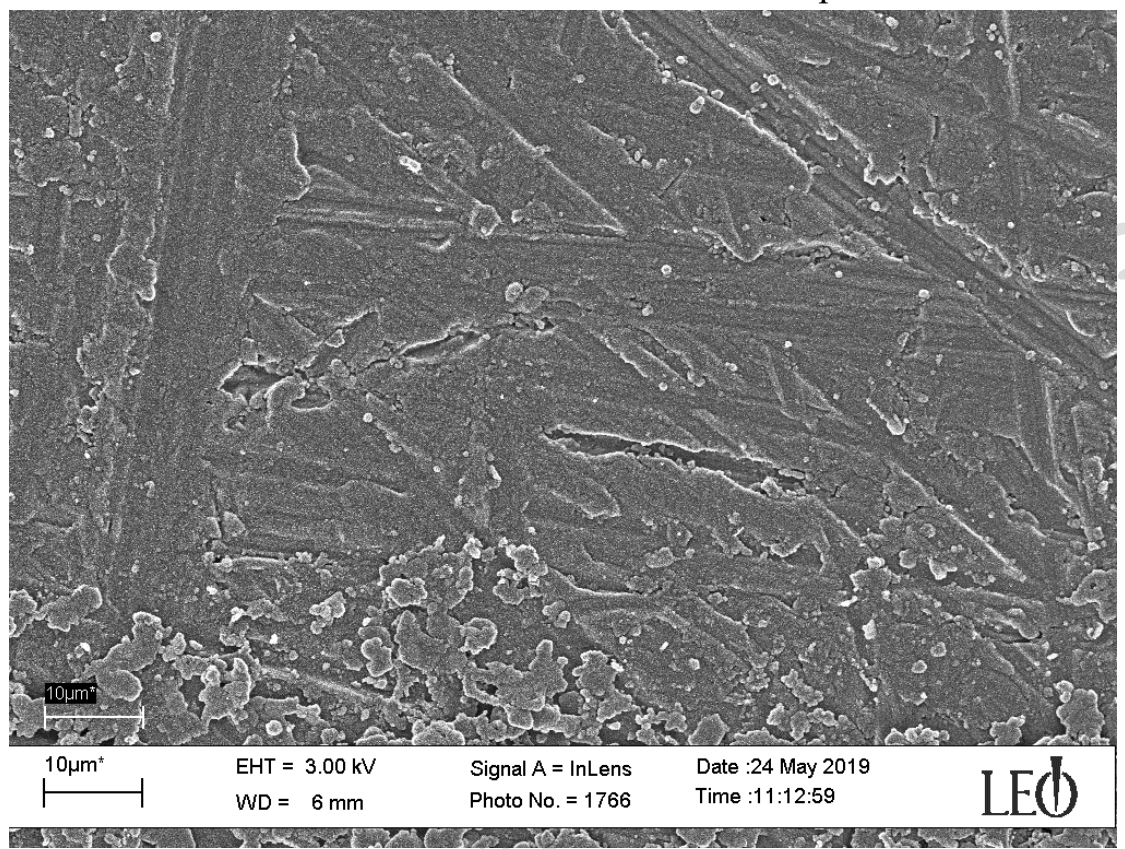

Figure (8) Second SEM image of graphene deposited on a $\mathrm{Cu}$ substrate 


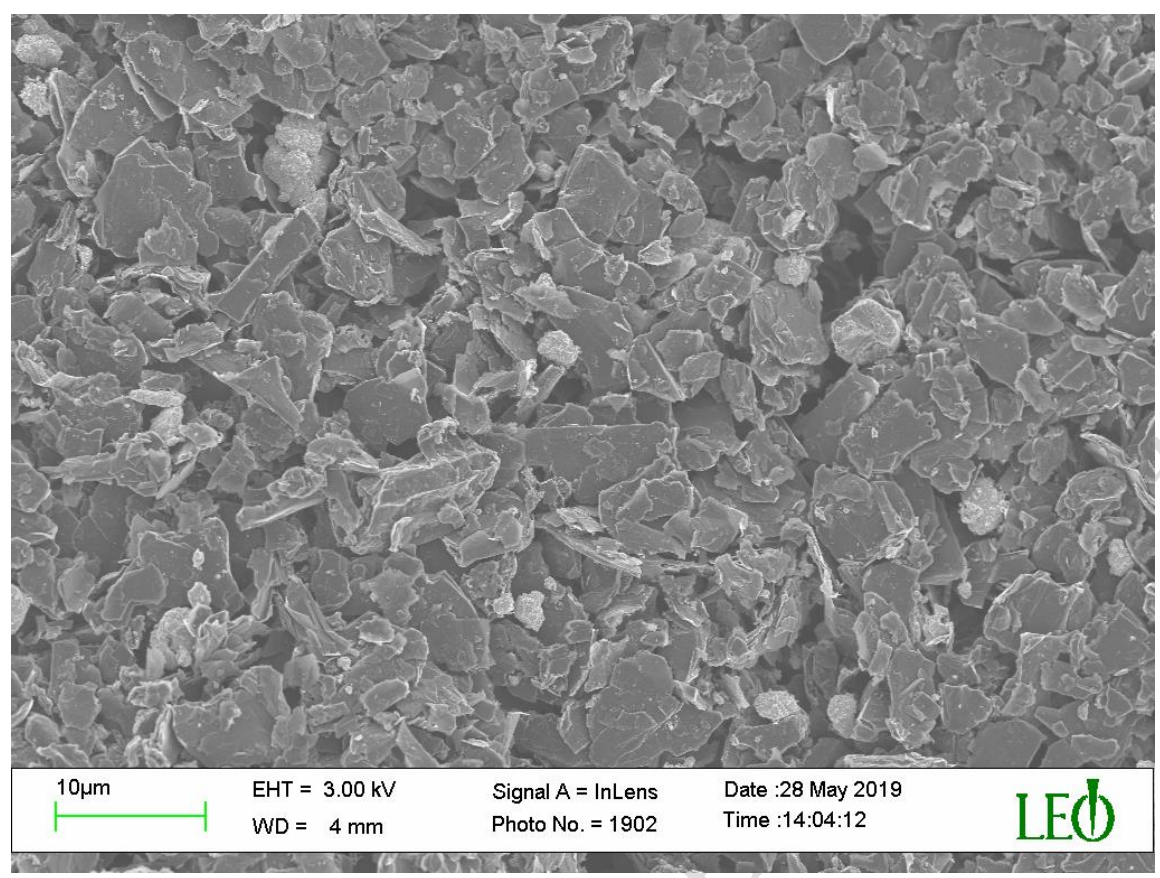

Figure (9) Second SEM image of graphene deposited on the $\mathrm{Cu}$ substrate coated by a slurry of Co nanoferrite

The SEM image in Figure (9) of the composite displays a 3D porous network with pore sizes in the range of micrometers. This network may be very useful for enhancing the electrochemical properties, as it not only provides an efficient pathway for electron transport but also reduces the ion diffusion resistance of the electrolyte for charge storage reactions. In addition, the 3D porous network provides abundant active sites to load $\mathrm{CoFe} 2 \mathrm{O} 4$ nanoparticles [19] 


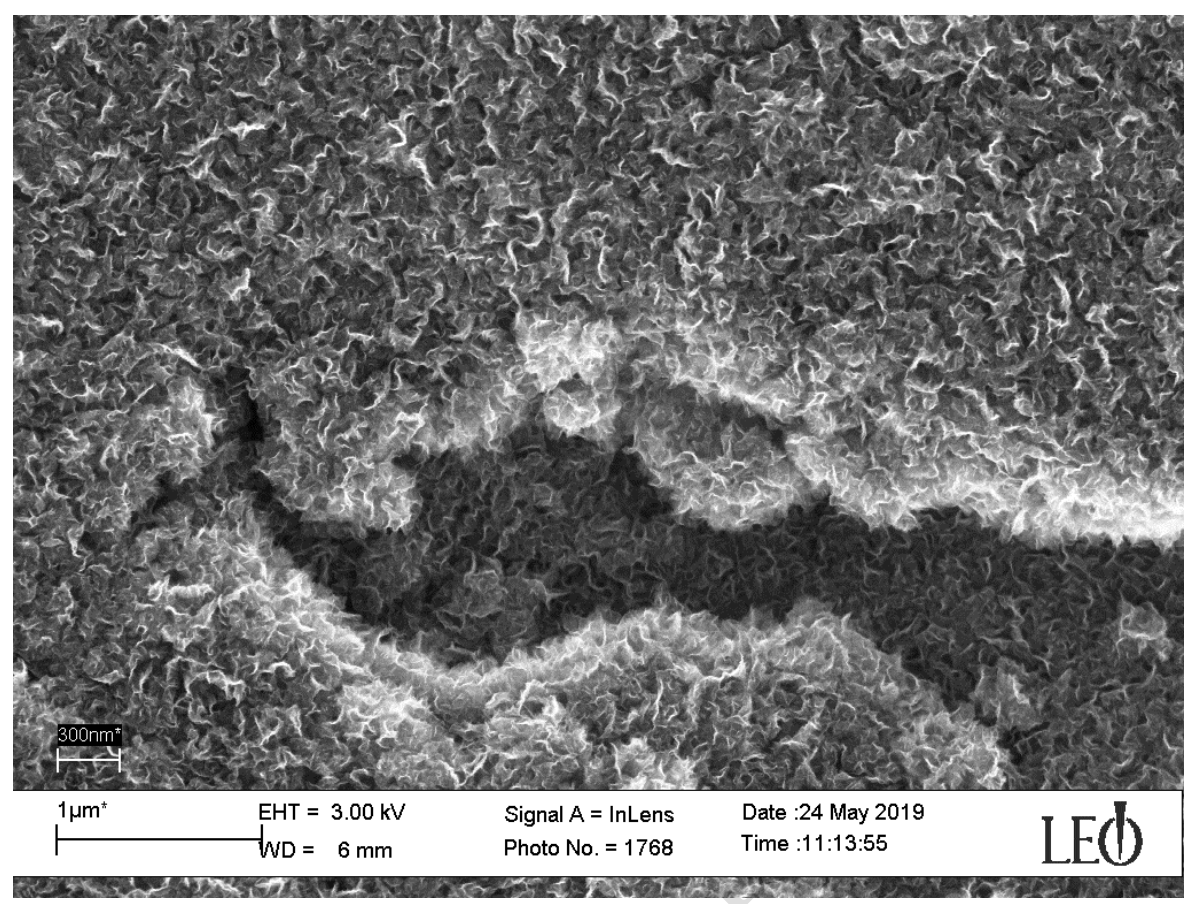

Figure (10) Third SEM image of graphene deposited on a $\mathrm{Cu}$ substrate

Finally, comparing Figure (10) and (11), Wrinkle-less surface of the graphene sheets seen in Figure (11) is a significant observation. This guarantees the properties of graphene prepared by the above mentioned method i.e. upon a a layer of Co nanoferrite slurry. Similar observations had been recorded in literature [20]; where a few layer graphene had been produced by magnetic nanoparticle assisted liquid phase exfoliation method. In the present work too the SEM images have confirmed the preparation of good quality graphene layers in the composite sample. 


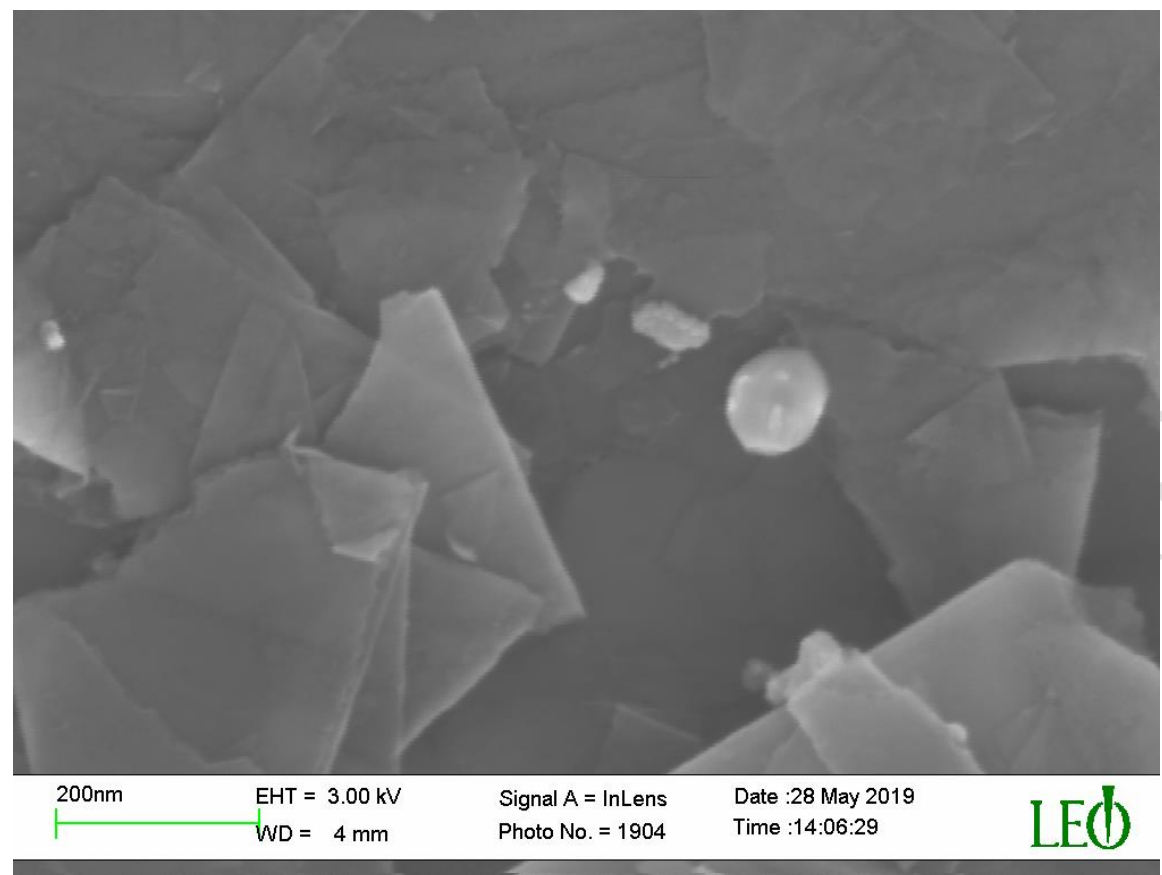

Figure (11) Third SEM image of graphene deposited on the $\mathrm{Cu}$ substrate coated by a slurry of Co nanoferrite

\section{Conclusions}

Graphene and graphene/ Co nanoferrite composite have been successfully synthesized via PECVD, on a $\mathrm{Cu}$ substrate and on a $\mathrm{Cu}$ substrate coated by a layer of slurry of Co nanoferrite The ferrite has caused an increase in the homogeneity of the surface area and improved its pore distribution. Our promising new method has coupled graphene with spinel ferrites which is supposed to produce an improved electrode for supercapacitors and/or batteries. A complete characterization of both the graphene and the composite sample via RAMAN Spectroscopy has been performed. Raman spectroscopy has proved a reliable technique in identification of the phase of the prepared samples and/or in differentiation between the graphene sample and the graphene nanoferrite composite. The SEM images has evidenced the supposed difference in the surface of graphene when deposited above a layer of Co nanoferrite; showing less defects, good wrinkle-less surface of graphene layers and better distributed pores.

\section{Acknowledgement}


Besides all members of the Materials Science and Electronics Lab, founded by Prof. Dr. Mostafa K. ElNimr ,in the Physics Department, Faculty of Science , Tanta University, the authors also acknowledge PMPWF, OEAD Austria, who has offered one of the authors, Shaimaa Ahmed habib the fund to go to Prof. Peter Knoll Lab in Austria.

\section{References}

1- Minjie Wang, Eui-Hyeok Yang, Nano-Structures \& Nano-Objects 15 (2018) 107-113

2- A. Hosseinzadeh , S. Bidmeshkipour, Y. Abdi , E. Arzi , S. Mohajerzadeh, Applied Surface Science 448 (2018) 71-77].

3- Tomo-o Terasawa, Koichiro Saiki, CARBON50 (2012) 869-874].

4- M.A. Darwish, S.A. Saafan, D. El- Kony, N.A. Salahuddin, Journal of Magnetism and Magnetic Materials 385 (2015) 99-106].

5- Bellad, S. S.; Watawe, S. C.; Shaikh, A. M.; Chougule, B. K.Bull. Mater. Sci.(2000),23,83].

6- F. Meng, M. Yang, L. Zhao, Y. Zhang, X. Shang, P. Jin, W. Zhang, A comparative study of the structural, magnetic and electrochemical properties of $\mathrm{Al3}+$ and $\mathrm{Cu} 2+$ substituted $\mathrm{NiZn}$ ferrite/reduced graphene oxide nanocomposites, Ceram. Int. 43 (2017) 15959-15964.

7- S. Supriya, S. Kumar and M. Kar, CFO-Graphene nano composite for High Performance Electrode Material, Mater. Today: Proc. 4 (2017) 5651-5656.

8- B. Bashir, W. Shaheen, M. Asghar, M. F. Warsi , M. A. Khan, S. Haider, I. Shakir, M. Shahid, Copper doped manganese ferrites nanoparticles anchored on graphene nano-sheets for high performance energy storage applications, J. Alloys Compd. 695 (2017) 881-887].

9- E. Talaie, P. Bonnick, X. Sun, Q. Pang, X. Liang, and L. F. Nazar, Methods and Protocols for Electrochemical Energy Storage Materials Research, Chem. Mater. 2017, 29, 90-105]. 
10- Moustafa A. Darwish, Alex V. Trukhanov, Oleg S. Senatov, Alexander T. Morchenko, Samia A. Saafan, Ksenia A. Astapovich, Sergei V. Trukhanov, Ekaterina L. Trukhanova, Andrey A. Pilyushkin , Antonio Sergio B. Sombra, Di Zhou , Rajshree B. Jotania and Charanjeet Singh, Nanomaterials 2020, 10, 492; doi:10.3390/nano10030492].

11- Rúbia Young Sun Zampiva, Claudir Gabriel Kaufmann Junior, Juliano Schorne Pinto, Priscila Chaves Panta, Annelise Kopp Alves, Carlos Pérez Bergmann Applied Surface Science 422 (2017) 321-330] .

12- P. Knoll ,J.Reynvaan ,M.Mayer, S. Surnev, P. Geistlinger ,K.Krenn, K.Ettinger, C. Prietln ,A.Hohenau, J. Gruenwald ,B.Kunert ,R.Resel Diamond \& Related Materials 65 (2016) 96-104].

13- Bhattacharyya, R., Prakash, O., Roy, S. et al. Graphene oxide-ferrite hybrid framework as enhanced broadband absorption in gigahertz frequencies. Sci Rep 9, 12111 (2019). https://doi.org/10.1038/s41598-019-48487-5.

14- Isaac Childres, Luis A. Jauregui, Wonjun Park, Helin Cao and Yong P. Chen .[Chapter 19] RAMAN SPECTROSCOPY OF GRAPHENE AND RELATED MATERIALS .

15- Rodolfo Morales Ibarra, Motonobu Goto, Juan García-Serna, Saida Mayela García Montes, Carbon Letters, https://doi.org/10.1007/s42823-020-00153-x].

16- Raghvendra Singh Yadav, Ivo Kuřitka, Jarmila Vilčáková, Miroslava Hajdúchová, August 2017, Advances in Natural Sciences: Nanoscience and Nanotechnology 8(4):045002, DOI: 10.1088/2043-6254/aa853a.

17- Yi Ding, Qingliang Liao, Shuo Liu, Huijing Guo, Yihui Sun, Guangjie Zhang \& Yue Zhang Scientific RepoRts | 6:32381 | DOI: 10.1038/srep32381] .

18- Rida Tabit, Othmane Amadine, Younes Essamlali, Karim D^anoun, Abdallah Rhihil and Mohamed Zahouily RSC Adv.,2018,8, 1351-1360.

19- Lingxia Zheng,ab Lingtong Guan,a Guang Yang,a Sanming Chenacand Huajun Zheng, RSC Adv., 2018, 8, 8607-8614].

20- Alireza Hadia, Jafar Zahirifara, Javad Karimi-Sabetb, Abolfazl Dastbaz, Ultrasonics Sonochemistry, Volume 44, June 2018, Pages 204-214]. 